\title{
Vulvodynia: a consideration of clinical and methodological research challenges and recommended solutions
}

This article was published in the following Dove Press journal:

Journal of Pain Research

9 October 2017

Number of times this article has been viewed

\author{
Serena Corsini-Munt ${ }^{1}$ \\ Kate M Rancourt ${ }^{\prime}$ \\ Justin P Dubél \\ Meghan A Rossi \\ Natalie O Rosen ${ }^{1,2}$ \\ 'Department of Psychology and \\ Neuroscience, ${ }^{2}$ Department of \\ Obstetrics and Gynecology, Dalhousie \\ University, Halifax, NS, Canada
}

\begin{abstract}
Vulvodynia, an idiopathic chronic vulvar pain, is a prevalent genital pain condition that results in significant impairment to sexual, relational, and psychological functioning of affected women and their romantic partners. Despite its high prevalence, there remain gaps in knowledge and health care access for women coping with vulvodynia, given its varied clinical presentation and no widely accepted treatment protocol. The past several decades have seen important advancements in understanding vulvodynia and developing effective treatments; however, progress has been impeded due to clinical and methodological challenges in conducting research with this vulnerable population. This review presents a brief overview of vulvodynia correlates, consequences, etiology, and treatment, and then turns its attention to considering the clinical and methodological challenges that hinder vulvodynia research. Identifying these barriers alongside potential mitigating solutions is essential to developing empirically supported treatments for all women affected by vulvodynia, across all age and minority groups. Potential solutions will require researchers to broaden eligibility criteria, examine subgroups of women, and expand definitions of treatment outcomes, and may be best facilitated by more active collaboration among research groups and across relevant disciplines. Engagement in these solutions may contribute to more representative findings and the development and dissemination of empirically based treatment options for this complex pain condition.
\end{abstract}

Keywords: female sexual pain, vulvodynia, provoked vestibulodynia, research barriers

\section{Background}

Vulvodynia refers to idiopathic, chronic vulvar pain lasting at least 3 months. ${ }^{1}$ Lifetime estimates of women of reproductive age indicate vulvodynia's prevalence at $8 \%-28 \%$ in the general population. ${ }^{2-6}$ The most common subtype of vulvodynia in premenopausal women is provoked vestibulodynia (PVD), which is characterized by pain upon contact to the vulvar vestibule and has a prevalence of $8 \%{ }^{5}$ Despite the high prevalence, only $60 \%$ of affected women seek treatment and only about $50 \%$ of those women receive a formal diagnosis of vulvodynia. ${ }^{5}$ Such findings highlight substantial gaps in knowledge and access to health care for women coping with this distressing pain condition.

Vulvodynia results in significant disruptions to the sexual, relational, and psychological functioning of affected women and their romantic partners, and these consequences can be just as distressing as the pain itself. ${ }^{7}$ The etiological pathways of vulvodynia and its associated impairments are multifactorial, and include biological, psychological, and interpersonal factors that modulate the experiences of individual women - from initial onset through treatment outcome - resulting in highly varied
Correspondence: Natalie O Rosen Department of Psychology and Neuroscience, Dalhousie University, 1355 Oxford Street, PO Box 15000 Halifax, NS B3H 4R2, Canada

Tel +l 9024944044

Fax +l 9024946585

Email nrosen@dal.ca 
clinical presentations and no widely accepted treatment strategy. ${ }^{7,8}$ Although great strides have been made in enhancing understanding of vulvodynia and developing effective treatments, there remain significant gaps in knowledge, further hindered by clinical and methodological challenges to conducting research with this highly vulnerable population. Identifying these challenges, coupled with a careful consideration of potential mitigating solutions, is essential to continued progress toward understanding the risk factors for vulvodynia and developing empirically supported treatment algorithms. This review begins with only a brief overview of the associated consequences of vulvodynia, proposed biopsychosocial etiological pathways, and current treatment approaches, referring readers to more comprehensive recent reviews for further details. We then turn our attention to current research challenges with this population - discussed in the context of clinical and methodological barriers - and potential solutions and directions for future study.

\section{Defining characteristics of vulvodynia}

According to the 2015 consensus terminology developed by the International Society for the Study of Vulvovaginal Disease, the International Society for the Study of Women's Sexual Health, and the International Pelvic Pain Society, persistent vulvar pain is classified into two categories: pain that is caused by a specific disorder (eg, pain resulting from squamous-cell carcinoma) and vulvodynia. ${ }^{1}$ Vulvodynia is characterized as vulvar pain with no clear identifiable cause, and may have associated features, such as inflammation, structural defects, and/or neuroproliferation. ${ }^{1}$ Vulvodynia is multifactorial, involving a complex collection of associated symptoms that include vulvar pain that is constant or periodic, provoked or spontaneous, and does or does not limit sexual intercourse. ${ }^{1,5}$ Women diagnosed with vulvodynia often describe the pain as pressure, burning, itching, stinging, irritation, stabbing, and/or rawness. ${ }^{9,10}$ Characteristics of pain presentation, such as eliciting situations and location, can be used further to categorize vulvodynia subtypes. ${ }^{1,7}$ For example, whereas unprovoked and diffuse vulvar pain is characteristic of generalized vulvodynia, pain that results from pressure localized to the vulvar vestibule defines PVD. ${ }^{7}$

\section{Comorbid conditions and consequences}

Vulvodynia is associated with several comorbid physical and psychological conditions, in addition to the financial, sexual, and relational consequences suffered by affected women and their partners. Various physical health symptoms (eg, insomnia, general weakness) are frequently reported in women with vulvodynia. ${ }^{11}$ In particular, women with vulvodynia are up to three times more likely than unaffected women to have one or more chronic pain conditions, including fibromyalgia, interstitial cystitis, painful bladder syndrome, irritable bowel syndrome, and temporomandibular disorder (ie, persistent facial pain). ${ }^{11-15}$ Women with both vulvodynia and a concomitant pain condition experience significantly greater depressive symptoms, trait anxiety, vulvar pain symptoms, and somatization than those with only one condition. ${ }^{14-16}$ In addition to comorbid pain conditions, the presence of spontaneous pain strongly differentiates vulvodynia subgroups and is predictive of greater physical and psychological difficulties than women with or without provoked pain and comorbid pain conditions. ${ }^{17}$

Depressive symptoms and anxiety disorders (eg, posttraumatic stress disorder) are more common among women with vulvodynia than women without this condition. ${ }^{6,16,18-20}$ Women with vulvodynia also report a number of other psychological concerns, including lower levels of happiness and poorer overall quality of life, compared to women without this pain condition. ${ }^{6,21,22}$ There is an economic burden associated with vulvodynia as well, since affected women tend to report more work and school absences, ${ }^{6}$ in addition to higher health care usage, than those who do not have vulvodynia. ${ }^{21}$

With its key interference being in the sexual relationship, women with vulvodynia experience a striking impact on their sexuality and more broadly their romantic relationships. Controlled studies have found that women with vulvodynia experience higher levels of sexual dysfunction and sexual distress, including difficulties with desire, arousal, orgasm, and satisfaction. ${ }^{23-25}$ Consequently, women report engaging in sexual activity less frequently, ${ }^{21}$ with some avoiding it altogether. ${ }^{24}$ Although overall levels of relationship satisfaction do not tend to differ from women without this pain, ${ }^{26}$ affected women report greater fears of losing their partners due to their pain, more emotional distance from their partners, and feelings of guilt and inadequacy as a romantic partner. ${ }^{27,28}$ Additionally, many women question their sense of womanhood because they experience pain during intercourse, and they express feelings of failure and shame related to not being able to satisfy their partners' sexual needs and pressure to maintain vaginal penetration as the main feature of sex in a heterosexual context. ${ }^{27}$ These consequences also extend to women's romantic partners, who are more likely to report sexual problems (eg, erectile difficulties) and poorer sexual satisfaction than controls, as well as a negative toll on their relationships due to the pain. ${ }^{29,30}$ Consequences of the pain may also act as maintaining factors, such that lower sexual 
desire and physiological arousal may potentially contribute to increased irritation and friction during penetrative sexual activities. Similarly, difficulties with sexual communication may contribute to unhelpful partner responses to pain and difficulty communicating when pain is present and severe. Overall, vulvodynia places a significant burden on many aspects of women's and partners' lives.

\section{Etiology}

Several biological, psychological, and interpersonal factors have been implicated in the development and maintenance of vulvodynia (see Pukall et al and Bergeron et al for more comprehensive reviews). ${ }^{7,31}$ There is evidence that both peripheral and central nervous system mechanisms contribute to the onset and persistence of vulvodynia. Biological factors thought to precipitate vulvodynia include atopic disease leading to vestibular mast-cell proliferation, ${ }^{32,33}$ vestibular mucosa alterations linked to combined hormonal contraception use ${ }^{34,35}$ (see also Reed et al), ${ }^{36}$ pelvic floor-muscle dysfunction, ${ }^{37,38}$ gene polymorphisms that interfere with pain regulation, ${ }^{39}$ and nociceptor proliferation and sensitization. ${ }^{40,41}$ Genetic predispositions to infection, inflammation, and/or hormonal changes have also been identified as risk factors for the development of vulvodynia. ${ }^{7}$ To date, the research methodology addressing biological factors has relied on retrospective and cross-sectional associations and animal models. Longitudinal and observational models may represent potential solutions to furthering our understanding of the biological factors leading to the development of vulvodynia.

Numerous psychological factors are also associated with an increased risk of vulvodynia. Antecedent depressive or anxiety disorders are correlated with a fourfold odds increase of vulvodynia among women in a community sample. ${ }^{42} \mathrm{~A}$ history of childhood maltreatment and victimization has consistently been associated with an increased risk of developing vulvodynia. ${ }^{43-45}$ A population-based study, for example, found that a history of physical and sexual abuse was associated with approximately fourfold- and sixfold-increased risk of vulvodynia, respectively; women with a history of combined childhood maltreatment, severe abuse, and poor familial support were 14 times more likely to develop vulvodynia than women with no history of maltreatment. ${ }^{43}$ Additional psychological factors linked to more pain and sexual impairment among women with vulvodynia include greater pain catastrophizing, hypervigilance to pain, fear avoidance, and lower pain self-efficacy and pain acceptance. ${ }^{46-48}$ With growing evidence for the role of romantic partners in chronic pain $^{49,50}$ and vulvodynia, ${ }^{51}$ studies have increasingly included partners in research examining biopsychosocial predictors of vulvodynia. Interpersonal variables, such as partner responses to the pain, intimacy, sexual goals, sexual communication, and attachment style, have been linked to the relational and sexual adjustment of both members of couples coping with vulvodynia, as well as women's pain experience. ${ }^{52-56}$ While many of the psychological and interpersonal factors identified as potential risk factors for vulvodynia stem from cross-sectional research and a lack of longitudinal research complicates understanding of specific underlying causes, there is mounting support for a multifactorial understanding of the etiology of vulvodynia.

\section{Treatment options}

At present, there is no gold-standard treatment guideline for women with vulvodynia, ${ }^{57}$ although several effective treatment options are available involving physical, medical, and psychological approaches (see Pukall et al, Bergeron et al, and Goldstein et al for more comprehensive recent reviews). ${ }^{31,58,59}$

\section{Pelvic floor physical therapy}

Based on evidence documenting significant dysfunction to the pelvic floor muscles among women with vulvodynia, ${ }^{60}$ many studies now demonstrate the efficacy of pelvic floor physical therapy. ${ }^{61}$ Physical therapy interventions, electromyography biofeedback, and electrical simulation have been found to reduce pain significantly during intercourse and enhance sexual function and quality of life following treatment. $^{62}$

\section{Surgical/medical}

According to a meta-analysis of 33 studies, the surgical intervention for vulvodynia - vulvar vestibulectomy - results in significant reductions in pain during intercourse in over $70 \%$ of treated women and significant improvements in sexual functioning. ${ }^{63}$ There are also several nonsurgical medical treatments for vulvodynia that target various hypothesized etiological mechanisms. These treatments include local anesthetics (lidocaine), botulinum toxin type A, antidepressants, anticonvulsants, hormonal treatments, and anti-inflammatory agents (eg, corticosteroids), with mixed evidence to support their use.$^{58}$ Although there is some evidence to suggest some efficacy for antidepressants, lidocaine, corticosteroids, and interferon, evidence from randomized clinical trials (RCTs) or lack thereof does not consistently support their use. Hormonal treatments (topical estradiol and testosterone), botulinum toxin type $\mathrm{A}$, and certain anti-inflammatory agents 
(cromolyn cream, enoxaparin, lysate of fetal fibroblasts) appear more promising and warrant continued study. Mixed evidence and methodological limitations (no randomization or controlled groups, small sample sizes, lacking data on complication rates) suggest the need for further research to evaluate the efficacy of these surgical and medical treatments.

\section{Psychological}

Psychological treatments are noninvasive and designed to target the multifaceted nature of the etiology of vulvodynia. In other words, psychological approaches address both pain management and sexual and relationship factors (eg, reduced desire, difficulties with sexual communication, restricted sexual repertoire, myths about sex) that may contribute to exacerbating the pain and associated sexual difficulties. Cognitive behavior therapy (CBT) has received the most empirical support, with studies revealing that CBT - delivered in individual or group format - results in greater improvements in pain and sexual function that are maintained up to 1 year later when compared to supportive psychotherapy ${ }^{64}$ and corticosteroids. ${ }^{65}$ In a clinical trial for women with PVD comparing vestibulectomy, CBT, and biofeedback, surgery resulted in greater posttreatment gains at 6-month followup. ${ }^{66}$ However, at the 2.5-year follow-up, there were no longer any differences in scores for pain during intercourse between CBT and vestibulectomy, such that treatment gains were maintained for both groups. ${ }^{67} \mathrm{~A}$ waitlist-controlled trial examining a mindfulness-based cognitive group-therapy program for women with PVD showed significant improvements in pain, sexual, and psychological outcomes compared to the control group. ${ }^{68}$ In a small-sample pilot study evaluating the efficacy of a 12-session couple-based CBT intervention for PVD, posttreatment outcomes showed significant improvements in pain and sexuality outcomes, in addition to improvements in psychological functioning for both women and their partners compared to pretreatment. ${ }^{69}$

\section{Multidisciplinary}

While the results achieved from individual therapies are notable, the multifactorial etiology of vulvodynia suggests that combining these approaches may be maximally beneficial. The limited available evidence from integrated medical, psychological, and physical therapy treatments suggests that women accrue significant reductions in their pain and improvements in sexual functioning from before to after treatment, and that gains can be maintained up to 3 years later. $^{70-73}$ Methodological limitations of these studies (eg, small sample sizes, retrospective designs, no randomization or control groups) limit conclusions about efficacy and indicate that larger RCTs are necessary to determine if these approaches confer gains above and beyond the individual intervention components.

\section{Clinical challenges and solutions in conducting vulvodynia research Vulvodynia: a problem for women or for couples?}

Researchers and clinicians alike face the challenge of conceptualizing vulvodynia as either a problem experienced by the woman or one experienced by the couple. This determination can have important consequences for decisions relating to study design, participant recruitment, and treatment. The dichotomy is also present among theoretical models of pain, with some models conceptualizing pain as a personal experience, such as the biomedical model of pain, versus models that consider pain within interpersonal systems, such as the empathy model of pain, communication models of pain, and the biopsychosocial model of pain. ${ }^{74-76}$ Despite strong research underscoring the interpersonal dimensions for women with vulvodynia and their partners, ${ }^{8,51,53,55}$ vulvodyniatreatment research has focused largely on the woman. The focus on pain and the pain patient in treatment research stems from the biomedical model of pain, ${ }^{77}$ the medicalization of sexual pain, ${ }^{78}$ and may also relate to the research-design challenges of including the partner and a dearth in interpersonal focus in sexuality research. ${ }^{79}$

However, psychological interventions and multimodal approaches (eg, concurrent medical and psychological treatments) have the latitude to address pain-related consequences and target clinical outcomes for both women and their partners. Recommendations to include the partner in pain-treatment protocols have been identified as beneficial to the person with pain and their spouse.$^{80}$ Specific to vulvodynia, preliminary research with a small sample (eight couples) suggested that including the partner in CBT for PVD was feasible and demonstrated improvements from before to after treatment for women's pain, as well as sexual, relational, and psychological adjustment for both members of the couple ${ }^{69}$ RCTs are required to determine the efficacy of couple-based approaches, including CBT, but also other promising psychological treatments, such as mindfulnessbased cognitive therapy. ${ }^{81}$

\section{What is an "effective" treatment?}

Another significant issue in vulvodynia-treatment research concerns the consideration of what constitutes an "effective" 
treatment. Like the broader field of chronic pain, ${ }^{82}$ a recent review demonstrated that many interventions for vulvodynia do not demonstrate strong efficacy in reducing the pain. ${ }^{58}$ Moreover, many treatments for vulvodynia reduce but do not eliminate women's vulvovaginal pain and associated sexual impairments. ${ }^{58}$ In line with biomedical models, it is not uncommon for vulvodynia interventions to be evaluated according to the capacity to reduce women's pain, which is the predominant symptom of vulvodynia, while other important domains of impairment (eg, psychological and sexual well-being) are not evaluated ${ }^{67}$ It is particularly important for clinicians to reinforce alternative treatment outcomes: given that many women and couples seek therapy seeking ways to achieve penetration, clinicians can also fall into this trap and reinforce the coital imperative belief by using penetration as a metric for treatment success. Therefore, psychotherapy options (eg, CBT, sex therapy) for women experiencing vulvodynia often recommend focusing on treatment outcomes independently of pain ratings and the ability to complete vaginal penetration, such as enhancing sexual pleasure, satisfaction, couple intimacy, and increasing pain self-efficacy. ${ }^{83}$ Indeed, alternative models, such as biopsychosocial models of vulvodynia, emphasize that improving functional capacity and reducing symptom-related distress are also essential markers of treatment efficacy. ${ }^{8,84}$ Recent IMMPACT (Initiative on Methods, Measurement, and Pain Assessment in Clinical Trials) guidelines on vulvodynia urge researchers to consider emotional function, quality of life, and sexual well-being as core measurement domains in clinical trials for vulvodynia. ${ }^{84}$ Several recent studies have pointed to other potentially important markers of treatment success, including reductions in sexual distress ${ }^{29}$ and fear-avoidance variables (eg, fear of pain, pain catastrophizing, pain hypervigilance), ${ }^{47}$ as well as increases in pain self-efficacy ${ }^{85}$ and pain acceptance. ${ }^{48}$ Therefore, while pain and sexual function remain the primary presenting problems that treatments aim to address, ${ }^{84}$ in future, researchers are encouraged to assess additional outcomes, as they may meaningfully capture the degree of improvement experienced by women undergoing treatment for vulvodynia. In so doing, researchers will enhance knowledge of novel treatment targets for clinicians, and thus may promote symptom adaptation in addition to symptom management.

\section{What is the "right" treatment and for whom?}

The complex, multifactorial etiology of vulvodynia suggests the potential for individual variability in the factors contributing to the onset and persistence of this condition and thus treatment targets. As such, Pukall et al ${ }^{7}$ suggested that identifying subgroups of affected women might be useful for improving treatment algorithms and successful outcomes, eg, some women may present with strong psychological and/or social features, whereas others may not. There are similar calls for more tailored approaches in the broader chronic-pain field. ${ }^{82}$ However, to direct women and/ or couples to appropriate treatment, it will be important to attend not only to the array of biomedical factors but also the psychosocial factors that are affecting their well-being and pain coping (see Bergeron et al for review). ${ }^{31}$ For example, biomedical factors demonstrating genetic links with inflammatory responses are being investigated in the search to develop new drug options that may be particularly helpful for women with genetic predispositions. ${ }^{86}$ Furthermore, it is possible that women who are partnered might benefit more from a relationship-focused treatment than one that is individually focused, and that women with stronger psychological features (eg, avoidance, psychological distress) might benefit more from psychological or multimodal treatments. Decisions regarding tailored approaches should be made within the context of understanding the sexual dysfunction that women with vulvodynia (and their partners) experience. Clinicians and researchers are urged to consider the needs and potential benefits for their patients beyond facilitating painfree intercourse. For example, the barometer for treatment success might be better set at achieving sexual pleasure and satisfaction through sexual activity other than intercourse, or by equipping couples with pain-management strategies that can be used in a sexual context.

Examining predictors of treatment success may be pivotal in identifying subgroups of women who may benefit maximally from particular interventions. However, very little is known about the factors that predict treatment efficacy in vulvodynia. Individual differences - such as high interpersonal distress regarding pain, low treatment expectancies, younger age, and less education - have been found to predict poorer treatment response to CBT for chronic pain more generally. ${ }^{87}$ In addition, greater pretreatment fear-avoidance beliefs have been shown to predict poorer treatment response (ie, less pain reduction) in both medical (eg, opioid) and psychological (eg, CBT) treatments for chronic pain. ${ }^{88,89}$ Similarly, in vulvodynia, women with lower avoidance and higher pain self-efficacy at pretreatment report lower pain and sexual dysfunction 6 months after topical corticosteroid treatment. In contrast, women with lower pretreatment fear of pain and pain catastrophizing, as well as higher pain self-efficacy, 
report lower pain at 6-month follow-up after receiving group CBT. ${ }^{90}$ Finally, Brown et al found that women with vulvodynia who reported more sexually satisfying relationships at pretreatment were three times more likely to have treatment success (ie, $\geq 50 \%$ reduction in pain during intercourse posttreatment) taking milnacipran, an antidepressant, than those with lower pretreatment sexual satisfaction. ${ }^{91}$ While the latter findings point to the importance of accounting for pretreatment factors, this preliminary study was conducted with a small sample of women $(n=22)$ and did not include a placebo-control group.

These preliminary and small number of findings show that the efficacy of a treatment may depend on the unique constellation of individual factors that they bring to the treatment environment. Therefore, understanding how demographic, psychological, physical, and relationship characteristics moderate treatment success is a necessary step in understanding which treatments will work for which women with vulvodynia. Importantly, studying treatment moderators may also help researchers and clinicians understand the "dosing" of particular interventions, ie, how much emphasis should be put on particular therapeutic interventions. For example, if women have strong baseline fear-avoidance beliefs, ${ }^{90}$ this may cue clinicians to be more attentive in intervening in these specific factors to increase the effectiveness of their interventions.

\section{What clinical factors complicate treatment access and efficacy?}

A final consideration of clinically relevant challenges to treatment research concerns understanding how complicating factors, such as geographical location, socioeconomic barriers, and comorbidities, may influence both access to participating in treatment trials and treatment effectiveness. Only $50 \%-60 \%$ of women with vulvodynia symptoms seek out diagnostic and treatment services, ${ }^{2,3}$ with perceived stigmatization playing an influential role in women's helpseeking behavior. ${ }^{92}$ Geographical barriers, time, and financial concerns may also influence the degree to which women seek care for this condition. Gynecologists, pelvic floor physical therapists, and psychologists are often situated in urban centers, meaning that the most effective treatment approaches are not readily available to women in rural areas. Moreover, the time-related (eg, missed work and travel) and financial costs of some interventions may be prohibitive, especially to those without insurance coverage for these health services. As such, researchers need to consider how to improve access to effective treatments for women with vulvodynia experiencing these barriers. Distance treatments are showing promising effects in diverse physical and mental health populations, ${ }^{93}$ including individuals with chronic pain ${ }^{94}$ and women with sexual dysfunction, ${ }^{95}$ and may warrant investigation for women with vulvodynia. Approaches to distance treatment span from online educational programs to moderated support groups to virtual or distance therapies facilitated by trained professionals, the latter of which provides the strongest evidence of equivalency with face-to-face psychological interventions. ${ }^{93}$

The etiological literature is clear that both physical and psychological comorbidities are common among women with vulvodynia (for review, see Pukall et al and Bergeron et al), ${ }^{7,31}$ yet women with significant comorbidities are often screened out of clinical treatment trials, ${ }^{64,65,96}$ in order to improve internal validity. Therefore, very little is known about how women with more complex presentations respond to the vulvodynia interventions that have received the most empirical support, such as CBT. Presumably, more complex presentations will require more flexible approaches to intervention, perhaps requiring deviations and/or modifications to treatment protocols that are deemed efficacious in the research literature. As the efficacy of interventions for women with vulvodynia and comorbid conditions is unknown, a multimodal treatment approach has the flexibility concurrently to address presenting symptoms of vulvodynia and the comorbid condition or to address symptoms in close succession. ${ }^{31,51,58}$ Understanding and addressing the impact of comorbidities in interventions for vulvodynia may help to improve access and treatment efficacy for women requiring more complicated interventions.

\section{Methodological challenges and solutions in conducting vulvodynia research \\ Volunteer bias}

As is the case in all research, vulvodynia research hinges on those women and couples who are willing to participate. However, vulvodynia research may be further limited by participants' willingness to disclose intimate and private details about the pain and their sexuality. Indeed, compared to those who do not volunteer for sex research, those that choose to participate report more sexual experience, more positive attitudes about sex, and less guilt about sex.${ }^{97}$ Unlike community participants, though, many women with vulvodynia report experiences of misdiagnosis and stigmatization, ${ }^{5,92}$ and may thus be highly motivated to contribute to improving treatment options and efficacy. 
Dyadic research in vulvodynia faces the additional hurdle of the partner being willing to participate alongside the woman experiencing pain. Partner unwillingness has been cited as a common reason for nonparticipation in research..$^{55}$ Couples who choose to participate may be distinct from those who do not in their openness to disclose private information. It is possible that they may already demonstrate a more teamlike approach to the pain (ie, viewing the pain as a couple problem) and better couple communication compared to couples uninterested or unwilling to participate in research together. More distressed women and couples may also be less likely to participate in etiological studies, which typically require participants to refrain from treatment during the study. Consequent to these potential biases, if participants are less distressed and more open about their sexuality, then it is possible that vulvodynia research is underestimating the severity of the pain and its associated consequences. Self-selection may be an inherent limitation in vulvodynia research, but it can be mitigated using randomization when appropriate (eg, in clinical trials), by assessing the presence of vulvar pain in population samples (to compare to research samples), and by focusing on wider recruitment efforts in the community (ie, not recruiting only from clinical referrals). Moreover, researchers are encouraged to collaborate with primary-care providers (eg, general practitioners) and expert consultants in tertiary-care settings to facilitate recruitment of women at varying stages in their health care-seeking journey.

\section{Representative sampling}

Many vulvodynia studies include women and couples who are predominantly white, young (ie, aged mid-20s to mid30 s on average), well educated (ie, have completed postsecondary education), and who identify as heterosexual and cisgender. ${ }^{64,92,98}$ This demographic makeup is problematic, given population-based research indicating that Hispanic women are $80 \%$ more likely to experience chronic vulvar pain compared to white and black women. ${ }^{3}$ Differences among women with vulvodynia from minority groups are currently unknown. There is only preliminary evidence pointing to differences between women who identify as heterosexual and lesbian, with heterosexual women reporting that the pain had a more negative effect on their relationship compared to lesbian women (75.3\% vs $52.2 \%) .{ }^{99}$ Improving the representativeness of vulvodynia sampling is all the more important, given that there are gaps in knowledge about physical health among sexual minorities, ${ }^{100}$ largely related to demonstrated patterns of excluding sexual-minority couples from research ${ }^{101}$ and clinical trials. ${ }^{102}$
Researchers often aim to recruit homogeneous samples of women with vulvodynia to control for confounding factors, such as hormonal influences (eg, physical and sexual changes associated with menopause) or comorbid conditions that may also influence the pain or the individual's functioning. While homogeneous samples allow researchers to compare between studies and test for intervention effects while controlling for confounding factors (ie, another treatment or health difficulty), the generalizability of findings is limited. Researchers should consider recruiting diverse samples across comorbid conditions, culture, sexual orientation, and age (eg, pre-, peri-, and postmenopausal women). Broadening inclusion criteria and the use of representative sampling can be promoted by targeting recruitment of diverse women and couples and using population-based sampling to address geographical limitations. The use of online-survey platforms, which eliminate the need to rely on local participants, may allow researchers to gather data from those living outside urban settings and to target more diverse samples, promoting the generalizability of research findings as a result. To address issues of comparison, researchers can track participant characteristics to allow for analyses to proceed with the whole sample and with subsamples when appropriate.

Given that pain is a primary feature of vulvodynia, it is no surprise that pain during intercourse is typically the primary outcome of interest. ${ }^{64,66,69,70,103}$ However, using pain during intercourse as the primary outcome requires that women are still engaging in activity that provokes pain. Many studies include only women and couples that have retained some sexual function by remaining sexually active, while women who are avoiding sexual activities - including those that trigger pain or otherwise - are excluded. Similarly, researchers often utilize eligibility-cutoff points in terms of the perceived pain intensity and the length of time since pain onset. ${ }^{64,66,104}$ Specifically, some studies only include women if their reported pain is greater than 4 out of 10 on a 10-point scale. ${ }^{103,105}$ Therefore, women who are in the early stages of experiencing pain, whose pain is of lower intensity - regardless of the level of distress and interference it causes - and those who are potentially the most impaired or distressed, as reflected by their heightened avoidance, may not be captured in prior research.

\section{Understanding mechanisms of psychological treatments}

The existing evidence on the efficacy of treatments for vulvodynia suggests that there is room for improvement. In addition to developing and evaluating novel treatments, 
another valuable approach may be to explore the mechanisms that contribute to treatment success for empirically supported treatments. All treatments for vulvodynia are developed based on theoretical mechanisms of action, ${ }^{58}$ and thus evaluating whether these mechanisms hold true will allow researchers and clinicians to focus on the aspects of treatments that contribute to change. For example, changes in fear-avoidance beliefs are a core component of the theoretical mechanism behind CBT for vulvodynia, ${ }^{66}$ whereas changes in interpersonal variables like sexual communication are an added core theoretical mechanism in couple-based CBT. ${ }^{69}$ Ultimately, the process of evaluating treatment mechanisms may allow treatments to be refined and/or expanded to increase their efficacy.

An array of research questions, designs, and statistical approaches would help build literature evaluating mechanisms of psychological treatment change in vulvodynia. These may include, but are not limited to examining trajectories of change over the course of therapy, evaluating whether theorized variables mediate treatment outcomes, and manipulating mediators to demonstrate that they lead to changes in treatment outcomes. ${ }^{106,107}$ As has been discussed by others, ${ }^{106,108,109}$ examining mechanisms of psychological treatments will require researchers to reconsider aspects of their research designs: most importantly, the inclusion of multiple measurement points over the course of therapy and posttreatment, in order to address the temporal precedence of mediators/mechanisms (ie, the critical notion that changes in the mediator must precede changes in the outcome). ${ }^{110}$ To date, no studies have examined treatment mechanisms in psychological interventions for vulvodynia.

\section{Dissemination of treatment research}

As more treatments are tested for efficacy, researchers must consider how to disseminate treatment protocols beyond publication in peer-reviewed journals. Innovative efforts should be made to share treatment protocols at academic and clinical conferences, within teaching hospitals, through clinical workshops, and online platforms. Developing treatment manuals that clearly outline effective protocols is an important step in the knowledge-translation process, as well as making these manuals available free or at low cost. Clear clinical trial protocols and treatment manuals will help ensure appropriate replication and adequate dissemination for trained clinicians to administer empirically validated treatments to women with vulvodynia and their partners. Equally important as the effective knowledge transfer of significant findings and their implications is a consideration of publi- cation bias, in which negative findings often go unshared. When possible, researchers should include negative findings in their publications to aid their colleagues and clinicians in understanding relevant and less relevant factors in the study and treatment of vulvodynia.

Given the centrality of psychoeducation in many treatment protocols, ${ }^{66,69,111}$ researchers may consider the development and testing of educational programs that could serve women in all geographical regions, even if those women do not have access to health care providers with specific expertise in vulvodynia. Such education programs could synthesize research findings for lay audiences, and be delivered as brief educational workshops online or within the community. ${ }^{111}$ Delivery platforms might include women's health-awareness days and women's health and gynecology websites, as well as those dedicated to vulvar health (eg, National Vulvodynia Association [www.nva.org]). Additionally, the development of curricula (eg, FAQ sheets, downloadable "webinars") detailing information about vulvodynia and genitopelvic pain for primary-care physicians has the potential to increase knowledge for diagnosis and treatment. Such interventions should be empirically based in their development, and tested for their efficacy using a rigorous design, such as an RCT.

\section{Conclusion}

Researchers and clinicians have made great progress in understanding the etiology and underlying pathophysiology of vulvodynia, illustrating the immense degree of heterogeneity in women suffering from this condition. ${ }^{7}$ By comparison, the treatment literature has fallen behind, further hindered by clinical and methodological research challenges identified in this review. Pukall et $\mathrm{al}^{59}$ suggested that a phenotypic approach to treatment may better serve the unique needs of subgroups of women with vulvodynia by allowing for the selection of treatments that target particular pathologies. Clinical trials are needed, however, to provide empirical support for this approach. In summary, and as reflected in Figure 1, the challenges to conducting research with this highly vulnerable and frequently misunderstood and neglected population include biased (largely homogeneous) samples that limit the generalizability of the findings, a narrow scope of treatment outcomes, and a lack of evidence to support treatment mechanisms. Possible solutions will require researchers to expand their eligibility criteria to examine subgroups of women within their studies, broaden their definitions of treatment outcomes, and call upon innovative ways of accessing and 


\section{Challenges}

To determine the efficacy of treatment approaches that address interpersonal aspects of vulvodynia (eg, partner inclusion in treatment, mindfulness therapy, multimodal treatment approaches)

Often, consequences and functioning that contribute to quality of life extend beyond the pain experience itself

Treatments are not "one size fits all", and certain approaches may benefit certain individuals/couples

Barriers to treatment access (eg, geographical location)

Little is known about treatment response in the presence of comorbid conditions

Volunteer bias in recruitment and participation

Low generalizability of study results because of homogeneous samples

Barriers to inclusion in research (eg, geographical location, minority populations)

Little is known about treatment mechanisms
$\begin{aligned} & \text { Low dissemination of treatment protocols and research } \\ & \text { findings that would facilitate diagnosis and treatment of } \\ & \text { vulvodynia }\end{aligned}$

Figure I Summary of challenges and recommendations

including women and couples who are not well integrated into the health care system. Collaboration across research groups - and across relevant health disciplines - may be the key to improving the size and representativeness of study samples, and ensuring that studies reflect the biopsychosocial complexity of vulvodynia.

\section{Acknowledgment}

The authors' research has been supported by an operating grant from the Canadian Institutes of Health Research (CIHR) awarded to Natalie O Rosen.

\section{Disclosure}

The authors report no conflicts of interest in this work.

\section{Recommendations}

Research methodology that includes randomized clinical trial design, including assessment of treatment predictors and potential moderators

Broaden treatment and research outcomes to include psychosexual, emotional, and interpersonal variables

Consider individual factors (eg, relationship status and psychological function) in addition to physical markers; evaluate potential moderators of treatment success (eg, psychological variables)

\section{Online and virtual treatment programs}

Examine the efficacy of multimodal treatment approaches that can address comorbidities in addition to vulvodynia and analyses including subsamples

Randomization when appropriate, use of populationrepresentative samples, and enhanced community-recruitment efforts

Broader recruitment to allow for comparison of diverse populations and analyses including subsamples

Use of online survey platforms to facilitate participation from diverse and nonlocal participants, targeted recruitment

Include multiple measurement points in clinical trials (beyond before and after), which would allow for the examination of trajectories of change of theorized mediators

Clear treatment protocols and manuals, online dissemination, and development and testing of educational programs

\section{References}

1. Bornstein J, Goldstein AT, Stockdale CK, et al. 2015 ISSVD, ISSWSH, and IPPS consensus terminology and classification of persistent vulvar pain and vulvodynia. $J$ Sex Med. 2016;13:607-612.

2. Reed BD, Harlow SD, Sen A, et al. Prevalence and demographic characteristics of vulvodynia in a population-based sample. Am J Obstet Gynecol. 2012;206:170.e1-e9.

3. Harlow BL, Stewart EG. A population-based assessment of chronic unexplained vulvar pain: have we underestimated the prevalence of vulvodynia? J Am Med Womens Assoc (1972). 2003;58:82-88.

4. Reed BD, Haefner HK, Sen A, Gorenflo D. Vulvodynia incidence and remission rates among adult women: a 2-year follow-up study. Obstet Gynecol. 2008;112:231-237.

5. Harlow BL, Kunitz CG, Nguyen RH, Rydell SA, Turner RM, MacLehose RF. Prevalence of symptoms consistent with a diagnosis of vulvodynia: population-based estimates from 2 geographic regions. Am J Obstet Gynecol. 2014;210:40.e1-e8.

6. Arnold LD, Bachmann GA, Rosen R, Rhoads GG. Assessment of vulvodynia symptoms in a sample of US women: a prevalence survey with a nested case control study. Am J Obstet Gynecol. 2007;196:128.e1-e6. 
7. Pukall CF, Goldstein AT, Bergeron S, et al. Vulvodynia: definition, prevalence, impact, and pathophysiological factors. J Sex Med. 2016;13:291-304.

8. Bergeron S, Rosen NO, Morin M. Genital pain in women: beyond interference with intercourse. Pain. 2011;152:1223-1225.

9. Reed BD, Legocki LJ, Plegue MA, Sen A, Haefner HK, Harlow SD. Factors associated with vulvodynia incidence. Obstet Gynecol. 2014;123:225-231.

10. Sadownik LA. Etiology, diagnosis, and clinical management of vulvodynia. Int J Womens Health. 2014;6:437-449.

11. Leusink P, Kaptheijns A, Laan E, van Boven K, Lagro-Janssen A. Comorbidities among women with vulvovaginal complaints in family practice. J Sex Med. 2016;13:220-225.

12. Reed BD, Harlow SD, Sen A, Edwards RM, Chen D, Haefner HK. Relationship between vulvodynia and chronic comorbid pain conditions. Obstet Gynecol. 2012;120:145-151.

13. Nguyen RH, Veasley C, Smolenski D. Latent class analysis of comorbidity patterns among women with generalized and localized vulvodynia: preliminary findings. J Pain Res. 2013;6:303-309.

14. Zolnoun DA, Rohl J, Moore CG, Perinetti-Liebert C, Lamvu GM, Maixner W. Overlap between orofacial pain and vulvar vestibulitis syndrome. Clin J Pain. 2008;24:187-191.

15. Bair E, Simmons E, Hartung J, Desia K, Maixner W, Zolnoun D. Natural history of comorbid orofacial pain among women with vestibulodynia. Clin J Pain. 2015;31:73-78.

16. Lester RA, Brotto LA, Sadownik LA. Provoked vestibulodynia and the health care implications of comorbid pain conditions. J Obstet Gynaecol Can. 2015;37:995-1005.

17. Reed BD, Plegue MA, Williams DA, Sen A. Presence of spontaneous pain and comorbid pain conditions identifies vulvodynia subgroups. J Low Genit Tract Dis. 2016;20:57-63.

18. Reed BD, Haefner HK, Punch MR, Roth RS, Gorenflo DW, Gillespie BW. Psychosocial and sexual functioning in women with vulvodynia and chronic pelvic pain: a comparative evaluation. $J$ Reprod Med. 2000;45:624-632.

19. Iglesias-Rios L, Harlow SD, Reed BD. Depression and posttraumatic stress disorder among women with vulvodynia: evidence from the population-based woman to woman health study. J Womens Health (Larchmt). 2015;24:557-562.

20. Masheb RM, Wang E, Lozano C, Kerns RD. Prevalence and correlates of depression in treatment-seeking women with vulvodynia. J Obstet Gynaecol. 2005;25:786-791.

21. Arnold LD, Bachmann GA, Rosen R, Kelly S, Rhoads GG. Vulvodynia: characteristics and associations with comorbidities and quality of life. Obstet Gynecol. 2006;107:617-624.

22. Nguyen RH, Ecklund AM, Maclehose RF, Veasley C, Harlow BL. Co-morbid pain conditions and feelings of invalidation and isolation among women with vulvodynia. Psychol Health Med. 2012;17:589-598.

23. Brauer M, ter Kuile MM, Laan E, Trimbos B. Cognitive-affective correlates and predictors of superficial dyspareunia. J Sex Marital Ther. 2009;35:1-24.

24. Masheb RM, Lozano-Blanco C, Kohorn EI, Minkin MJ, Kerns RD. Assessing sexual function and dyspareunia with the Female Sexual Function Index (FSFI) in women with vulvodynia. J Sex Marital Ther. 2004;30:315-324.

25. Sutton KS, Pukall CF, Chamberlain S. Pain ratings, sensory thresholds, and psychosocial functioning in women with provoked vestibulodynia. $J$ Sex Marital Ther. 2009;35:262-281.

26. Smith KB, Pukall CF. A systematic review of relationship adjustment and sexual satisfaction among women with provoked vestibulodynia. J Sex Res. 2011;48:166-191.

27. Ayling K, Ussher JM. "If sex hurts, am I still a woman?" The subjective experience of vulvodynia in hetero-sexual women. Arch Sex Behav. 2008;37:294-304.

28. Elmerstig E, Wijma B, Bertero C. Why do young women continue to have sexual intercourse despite pain? J Adolesc Health. 2008;43:357-363.
29. Pazmany E, Bergeron S, Verhaeghe J, Van Oudenhove L, Enzlin P. Sexual communication, dyadic adjustment, and psychosexual wellbeing in premenopausal women with self-reported dyspareunia and their partners: a controlled study. J Sex Med. 2014;11:1786-1797.

30. Smith KB, Pukall CF. Sexual function, relationship adjustment, and the relational impact of pain in male partners of women with provoked vulvar pain. J Sex Med. 2014;11:1283-1293.

31. Bergeron S, Corsini-Munt S, Aerts L, Rancourt K, Rosen NO. Female sexual pain disorders: a review of the literature on etiology and treatment. Curr Sex Health Rep. 2015;7:159-169.

32. Bornstein J, Goldschmid N, Sabo E. Hyperinnervation and mast cell activation may be used as histopathologic diagnostic criteria for vulvar vestibulitis. Gynecol Obstet Invest. 2004;58:171-178.

33. Landry J, Martinov T, Mengistu H, et al. Repeated hapten exposure induces persistent tactile sensitivity in mice modeling localized provoked vulvodynia. PLoS One. 2017;12:e0169672.

34. Goldstein AT, Belkin ZR, Krapf JM, et al. Polymorphisms of the androgen receptor gene and hormonal contraceptive induced provoked vestibulodynia. J Sex Med. 2014;11:2764-2771.

35. Harlow BL, Vitonis AE, Stewart EG. Influence of oral contraceptive use on the risk of adult-onset vulvodynia. J Reprod Med. 2008;53:102-110.

36. Reed BD, Harlow SD, Legocki LJ, et al. Oral contraceptive use and risk of vulvodynia: a population-based longitudinal study. BJOG. 2013;120:1678-1684.

37. Morin M, Binik YM, Bourbonnais D, Khalifé S, Ouellet S, Bergeron $\mathrm{S}$. Heightened pelvic floor muscle tone and altered contractility in women with provoked vestibulodynia. J Sex Med. 2017;14:592-600.

38. Zolnoun D, Hartmann K, Lamvu G, As-Sanie S, Maixner W, Steege J. A conceptual model for the pathophysiology of vulvar vestibulitis syndrome. Obstet Gynecol Surv. 2006;61:395-401.

39. Heddini U, Bohm-Starke N, Grönbladh A, Nyberg F, Nilsson KW, Johannesson U. Serotonin receptor gene (5HT-2A) polymorphism is associated with provoked vestibulodynia and comorbid symptoms of pain. J Sex Med. 2014;11:3064-3071.

40. Bohm-Starke N, Hilliges M, Brodda-Jansen G, Rylander E, Torebjörk E. Psychophysical evidence of nociceptor sensitization in vulvar vestibulitis syndrome. Pain. 2001;94:177-183.

41. Pukall CF, Binik YM, Khalifé S, Amsel R, Abbot FV. Vestibular tactile and pain thresholds in women with vulvar vestibulitis syndrome. Pain. 2000;96:163-175.

42. Khandker M, Brady SS, Vitonis AF, MacLehose RF, Stewart EG, Harlow BL. The influence of depression and anxiety on risk of adult onset vulvodynia. J Womens Health (Larchmt). 2011;20:1445-1451.

43. Harlow BL, Stewart EG. Adult-onset vulvodynia in relation to childhood violence victimization. Am J Epidemiol. 2005;161:871-880.

44. Khandker M, Brady SS, Stewart EG, Harlow BL. Is chronic stress during childhood associated with adult-onset vulvodynia? J Womens Health (Larchmt). 2014;23:649-656.

45. Landry T, Bergeron S. Biopsychosocial factors associated with dyspareunia in a community sample of adolescent girls. Arch Sex Behav. 2011;40:877-889.

46. Desrochers G, Bergeron S, Landry T, Jodoin M. Do psychosexual factors play a role in the etiology of provoked vestibulodynia? A critical review. J Sex Marital Ther. 2008;34:198-226.

47. Desrochers G, Bergeron S, Khalifé S, Dupuis MJ, Jodoin M. Fear avoidance and self-efficacy in relation to pain and sexual impairment in women with provoked vestibulodynia. Clin J Pain. 2009;25:520-527.

48. Boerner KE, Rosen NO. Acceptance of vulvovaginal pain in women with provoked vestibulodynia and their partners: associations with pain, psychological, and sexual adjustment. J Sex Med. 2015; $12: 1450-1462$

49. Leonard MT, Cano A, Johansen AB. Chronic pain in a couples context: a review and integration of theoretical models and empirical evidence. J Pain. 2006;7:377-390.

50. Stark Taylor S, Davis MC, Zautra AJ. Relationship status and quality moderate daily pain-related changes in physical disability, affect, and cognitions in women with chronic pain. Pain. 2013;154:147-153. 
51. Rosen NO, Rancourt K, Corsini-Munt S, Bergeron S. Beyond a "woman's problem": the role of relationship processes in genital pain. Curr Sex Health Rep. 2014;6:1-10.

52. Rancourt KM, Flynn M, Bergeron S, Rosen NO. It takes two: sexual communication patterns and the sexual and relational adjustment of couples coping with provoked vestibulodynia. J Sex Med. 2017; 14:434-443.

53. Rosen NO, Bergeron S, Sadikaj G, Glowacka M, Delisle I, Baxter M. Impact of partner responses on sexual function in women with vulvodynia and their partners: a dyadic daily experience study. Health Psychol. 2014;33:823-831.

54. Rosen NO, Muise A, Bergeron S, Impett EA, Boudreau G. Approach and avoidance sexual goals in couples with provoked vestibulodynia: associations with sexual, relational, and psychological well-being. J Sex Med. 2015;12:1781-1790.

55. Bois K, Bergeron S, Rosen N, Mayrand MH, Brassard A, Sadikaj G. Intimacy, sexual satisfaction, and sexual distress in vulvodynia couples: an observational study. Health Psychol. 2016;35:531-540.

56. Rosen NO, Bois K, Mayrand MH, Vannier S, Bergeron S. Observed and perceived disclosure and empathy are associated with better relationship adjustment and quality of life in couples coping with vulvodynia. Arch Sex Behav. 2016;45:1945-1956.

57. Lua LL, Hollette Y, Parm P, Allenback G, Dandolu V. Current practice patterns for management of vulvodynia in the United States. Arch Gynecol Obstet. 2017;295:669-674.

58. Goldstein AT, Pukall CF, Brown C, Bergeron S, Stein A, KelloggSpadt S. Vulvodynia: assessment and treatment. J Sex Med. 2016;13: 572-590.

59. Pukall CF, Mitchell LS, Goldstein AT. Non-medical, medical, and surgical approaches for the treatment of provoked vestibulodynia. Curr Sex Health Rep. 2016;8:240-248.

60. Rosenbaum TY. Physical therapy management and treatment of sexual pain disorders. In: Leiblum SR, editor. Principles and Practice of Sex Therapy. 4th ed. New York: Guilford Press; 2007:157-177.

61. Bergeron S, Morin M, Lord MJ. Integrating pelvic floor rehabilitation and cognitive-behavioural therapy for sexual pain: what have we learned and were do we go from here? Sex Relation Ther. 2010;25:289-298.

62. Morin M, Carroll MS, Bergeron S. Systematic review of the effectiveness of physical therapy modalities in women with provoked vestibulodynia. Sex Med Rev. 2017;5:295-322.

63. Tommola P, Unkila-Kallio L, Paavonen J. Surgical treatment of vulvar vestibulitis: a review. Acta Obstet Gynecol Scand. 2010;89:1385-1395.

64. Masheb RM, Kerns RD, Lozano C, Minkin MJ, Richman S. A randomized clinical trial for women with vulvodynia: cognitive-behavioral therapy vs. supportive psychotherapy. Pain. 2009;141:31-40.

65. Bergeron S, Khalifé S, Dupuis MJ, McDuff P. A randomized clinical trial comparing group cognitive-behavioral therapy and a topical steroid for women with dyspareunia. J Consult Clin Psychol. 2016;84:259-268.

66. Bergeron S, Binik YM, Khalifé S, et al. A randomized comparison of group cognitive-behavioral therapy, surface electromyographic biofeedback, and vestibulectomy in the treatment of dyspareunia resulting from vulvar vestibulitis. Pain. 2001;91:297-306.

67. Landry T, Bergeron S, Dupuis MJ, Desrochers G. The treatment of provoked vestibulodynia: a critical review. Clin J Pain. 2008;24:155-171.

68. Brotto LA, Basson R, Smith KB, Driscoll M, Sadownik L. Mindfulness-based group therapy for women with provoked vestibulodynia. Mindfulness (NY). 2015;6:417-432.

69. Corsini-Munt S, Bergeron S, Rosen NO, Mayrand MH, Delisle I. Feasibility and preliminary effectiveness of a novel cognitive-behavioral couple therapy for provoked vestibulodynia: a pilot study. J Sex Med. 2014;11:2515-2527.

70. Brotto LA, Yong P, Smith KB, Sadownik LA. Impact of a multidisciplinary vulvodynia program on sexual functioning and dyspareunia. J Sex Med. 2015;12:238-247.
71. Sadownik LA, Seal BN, Brotto LA. Provoked vestibulodynia: women's experience of participating in a multidisciplinary vulvodynia program. J Sex Med. 2012;9:1086-1093.

72. Munday P, Buchan A, Ravenhill G, Wiggs A, Brooks F. A qualitative study of women with vulvodynia - II: response to a multidisciplinary approach to management. J Reprod Med. 2007;52:19-22.

73. Spoelstra SK, Dijkstra JR, van Driel MF, Schultz WC. Long-term results of an individualized, multifaceted, and multidisciplinary therapeutic approach to provoked vestibulodynia. J Sex Med. 2011;8:489-496.

74. Goubert L, Craig KD, Vervoort T, et al. Facing others in pain: the effects of empathy. Pain. 2005;118:285-288.

75. Hadjistavropoulos T, Craig KD, Duck S, et al. A biopsychosocial formulation of pain communication. Psychol Bull. 2011;137:910-939.

76. Sullivan MJ, Thorn B, Haythornthwaite JA, et al. Theoretical perspectives on the relation between catastrophizing and pain. Clin J Pain. 2001;17:52-64.

77. Dewitte M, Van Lankveld J, Crombez G. Understanding sexual pain: a cognitive-motivational account. Pain. 2011;152:251-253.

78. Farrell J, Cacchioni T. The medicalization of women's sexual pain. J Sex Res. 2012;49:328-336.

79. Dewitte M. On the interpersonal dynamics of sexuality. J Sex Marital Ther. 2014;40:209-232.

80. Keefe FJ, Rumble ME, Scipio CD, Giordano LA, Perri LM. Psychological aspects of persistent pain: current state of the science. J Pain. 2004;5:195-211.

81. Brotto LA, Basson R, Carlson M, Zhu C. Impact of an integrated mindfulness and cognitive behavioral treatment for provoked vestibulodynia (IMPROVED): a qualitative study. Sex Relation Ther. 2013; 28:3-19.

82. Borsook D, Kalso E. Transforming pain medicine: adapting to science and society. Eur J Pain. 2013;17:1109-1125.

83. Bergeron S, Rosen NO, Corsini-Munt S. Painful sex. In: Levine SB, Risen CB, Althof SE, editors. Handbook of Clinical Sexuality for Mental Health Professionals. 3rd ed. New York: Brunner-Routledge; 2016.

84. Pukall CF, Bergeron S, Brown C, Bachmann G, Wesselmann U. Recommendations for self-report outcome measures in vulvodynia clinical trials. Clin J Pain. 2017;33:756-765.

85. Davis SN, Bergeron S, Bois K, Sadikaj G, Binik YM, Steben M. A prospective 2-year examination of cognitive and behavioral correlates of provoked vestibulodynia outcomes. Clin J Pain. 2015;31: 333-341.

86. Foster DC, Falsetta ML, Woeller CF, et al. Site-specific mesenchymal control of inflammatory pain to yeast challenge in vulvodynia-afflicted and pain-free women. Pain. 2015;156:386-396.

87. Broderick JE, Keefe FJ, Schneider S, et al. Cognitive behavioral therapy for chronic pain is effective, but for whom? Pain. 2016;157: 2115-2123.

88. Grosen K, Olesen AE, Gram M, et al. Predictors of opioid efficacy in patients with chronic pain: a prospective multicenter observational cohort study. PLoS One. 2017;12:e0171723.

89. Wertli MM, Rasmussen-Barr E, Held U, Weiser S, Bachmann LM, Brunner F. Fear-avoidance beliefs-a moderator of treatment efficacy in patients with low back pain: a systematic review. Spine J. 2014; 14:2658-2678.

90. Desrochers G, Bergeron S, Khalifé S, Dupuis MJ, Jodoin M. Provoked vestibulodynia: psychological predictors of topical and cognitive-behavioral treatment outcome. Behav Res Ther. 2010;48: 106-115.

91. Brown C, Bachmann G, Foster D, Rawlinson L, Wan J, Ling F. Milnacipran in provoked vestibulodynia: efficacy and predictors of treatment success. J Low Genit Tract Dis. 2015;19:140-144.

92. Nguyen RH, Turner RM, Rydell SA, Maclehose RF, Harlow BL. Perceived stereotyping and seeking care for chronic vulvar pain. Pain Med. 2013;14:1461-1467.

93. Andersson G. Internet-delivered psychological treatments. Annu Rev Clin Psychol. 2016;12:157-179. 
94. Dear BF, Gandy M, Karin E, et al. The pain course: a randomised controlled trial examining an Internet-delivered pain management program when provided with different levels of clinician support. Pain. 2015;156:1920-1935.

95. Jones LM, McCabe MP. The effectiveness of an Internet-based psychological treatment program for female sexual dysfunction. $J$ Sex Med. 2011;8:2781-2792.

96. Morin M, Dumoulin C, Bergeron S, et al. Randomized clinical trial of multimodal physiotherapy treatment compared to overnight lidocaine ointment in women with provoked vestibulodynia: design and methods. Contemp Clin Trials. 2016;46:52-59.

97. Strassberg DS, Lowe K. Volunteer bias in sexuality research. Arch Sex Behav. 1995;24:369-382.

98. Ponte M, Klemperer E, Sahay A, Chren MM. Effects of vulvodynia on quality of life. J Am Acad Dermatol. 2009;60:70-76.

99. Blair KL, Pukall CF, Smith KB, Cappell J. Differential associations of communication and love in heterosexual, lesbian, and bisexual women's perceptions and experiences of chronic vulvar and pelvic pain. J Sex Marital Ther. 2015;41:498-524.

100. Lick DJ, Durso LE, Johnson KL. Minority stress and physical health among sexual minorities. Perspect Psychol Sci. 2013;8:521-548.

101. Andersen JP, Zou C. Exclusion of sexual minority couples from research. Health Sci J. 2015;9:10.

102. Egleston BL, Dunbrack RL Jr, Hall MJ. Clinical trials that explicitly exclude gay and lesbian patients. $N$ Engl J Med. 2010;362:1054-1055.
103. Corsini-Munt S, Bergeron S, Rosen NO, et al. A comparison of cognitive-behavioral couple therapy and lidocaine in the treatment of provoked vestibulodynia: study protocol for a randomized clinical trial. Trials. 2014;15:506.

104. Lindström S, Kvist LJ. Treatment of provoked vulvodynia in a Swedish cohort using desensitization exercises and cognitive behavioral therapy. BMC Womens Health. 2015;15:108.

105. Foster DC, Kotok MB, Huang LS, et al. Oral desipramine and topical lidocaine for vulvodynia: a randomized controlled trial. Obstet Gynecol. 2010;116:583-593.

106. Kazdin AE. Understanding how and why psychotherapy leads to change. Psychother Res. 2009;19:418-428.

107. Kraemer HC, Wilson GT, Fairburn CG, Agras WS. Mediators and moderators of treatment effects in randomized clinical trials. Arch Gen Psychiatry. 2002;59:877-883.

108. Kazdin AE. Mediators and mechanisms of change in psychotherapy research. Annu Rev Clin Psychol. 2007;3:1-27.

109. Laurenceau JP, Hayes AM, Feldman GC. Some methodological and statistical issues in the study of change processes in psychotherapy. Clin Psychol Rev. 2007;27:682-695.

110. MacKinnon DP, Fairchild AJ, Fritz MS. Mediation analysis. Annu Rev Psychol. 2007;58:593-614.

111. Brotto LA, Sadownik L, Thomson S. Impact of educational seminars on women with provoked vestibulodynia. JObstet Gynaecol Can. 2010;32: $132-138$.
Journal of Pain Research

\section{Publish your work in this journal}

The Journal of Pain Research is an international, peer reviewed, open access, online journal that welcomes laboratory and clinical findings in the fields of pain research and the prevention and management of pain. Original research, reviews, symposium reports, hypothesis formation and commentaries are all considered for publication.

\section{Dovepress}

The manuscript management system is completely online and includes a very quick and fair peer-review system, which is all easy to use. Visit http://www.dovepress.com/testimonials.php to read real quotes from published authors. 